\title{
OJO SECO Y SU IMPLICACIÓN EN LA FUNCIÓN VISUAL
}

\section{DRY EYE DISEASE AND ITS IMPLICATIONS ON VISUAL FUNCTION}

\author{
DOGRU $\mathrm{M}^{1}$, KAIDO $\mathrm{M}^{2}$, MATSUMOTO $\mathrm{Y}^{2}$, TSUBOTA K ${ }^{2}$
}

Uno de los cambios más importantes que tuvieron lugar durante las reuniones del grupo de ojo seco desde 2004 a 2006 fue el hecho de que la definición de ojo seco incluyera los trastornos de la función visual, a diferencia de la definición del grupo de ojo seco del Instituto Oftalmológico Nacional de 1994-1995.

Brevemente, la definición previa afirmaba que el ojo seco era un trastorno de la película lagrimal debido a un déficit de producción lagrimal o a una excesiva evaporación que provocaba una lesión de la superficie ocular interpalpebral asociada a síntomas de molestia ocular.

La actual definición describe el ojo seco como: «el ojo seco es una enfermedad multifactorial de la lágrima y de la superficie ocular que provoca síntomas de malestar, trastorno visual e inestabilidad de la película lagrimal, con potencial lesión de la superficie ocular. Se acompaña de un aumento de la osmolaridad de la película lagrimal y de la inflamación de la superficie ocular» (1).

La inclusión de la alteración visual en la definición del ojo seco se debe a la evidencia acumulada de que el ojo seco está realmente asociado con cambios visuales dinámicos. A pesar de que los tests estándares de medición de la agudeza visual (AV) son un método excelente de medir un aspecto de la función visual, la medición de la sensibilidad al contraste y del trastorno por luz («glare») proporciona información mucho más importante y detallada en aspectos específicos de la función visual. Recientemente, se ha publicado que la medición de la agudeza visual funcional (AVF) es un método importante de definir la «función visual detallada» (1). Este método ha demostrado ser eficaz en la detección de una «lesión oculta de la función visual» en pacientes con ojo seco que se quejaban de disminución de AV a pesar de presentar resultados normales en los exámenes de AV convencionales.

El sistema de Medición de la Agudeza Visual Funcional (Nidek, Japón) está actualmente disponible sólo en Japón y se utiliza para examinar el cambio a lo largo del tiempo en la agudeza visual continua. Las mediciones se inician desde la línea basal de la AV con su mejor corrección Landolt convencional, en cada sujeto.

Los optotipos se colocan a una distancia de 2,5 m en todos los pacientes, con una línea basal de AV mejor corregida mayor o igual a 20/200. Cada optotipo de Landolt se presenta a un determinado nivel de AV dentro del dispositivo que subtiende un ángulo equivalente al del optotipo del mismo nivel de $\mathrm{AV}$ presentado a una distancia de $5 \mathrm{~m}$ durante la medición de AV convencional Landolt. El tiempo de presentación de un optotipo es ajustable y los optotipos se cambian automáticamente dentro del rango de tiempo de presentación previamente establecido cuando el paciente responde al optotipo presentado.

Los pacientes delinean la orientación de los anillos de Landolt presentados automáticamente mediante el movimiento del mando desde la línea basal de la AV mejor corregida del inicio. Los anillos de Landolt aumentan de tamaño automáticamente cuando la respuesta es incorrecta. Si el anillo de Landolt es reconocido correctamente, el mismo tamaño de anillo se presenta de forma aleatoria otra vez. La AV durante 60 segundos es registrada como medida de la AVF (2).

\footnotetext{
${ }^{1}$ Facultad de Medicina de la Universidad de Keio, Departamento de Superficie Ocular y Óptica, Tokyo, Japón. Colegio de Odontología de Tokyo, Hospital de Ichikawa, Departamento de Oftalmología, Chiba, Japón

E-mail: muratodooru@yahoo.com

2 Facultad de Medicina de la Universidad de Keio, Departamento de Oftalmología, Tokyo, Japón
} 
Se ha sugerido que la medición de la AVF es un indicador importante de la capacidad de un sujeto en relación con ciertas actividades diarias tales como conducir, leer y hacer trabajos de ordenador (2).

Goto et al. ya habían publicado anteriormente anomalías de la AVF en sujetos con ojo seco (2). La AVF también se ha mostrado eficaz en la evaluación de los cambios dinámicos en la función visual en los ojos secos tras LASIK (3). Ishida et al. también comunicaron que la AVF en los ojos secos era significativamente menor que la de los sujetos controles y que, tras la inserción de un tapón en el punto lagrimal, mejoraba significativamente (4).

Un reciente estudio ha comunicado que la AVF de 10 segundos muestra correlación con la opacidad corneal y la presencia de vasos corneales en la mayoría de los pacientes con síndrome de StevensJohnson (SSJ). Sin embargo, no se encontró ninguna relación significativa entre los valores de AVF basal y la conjuntivalización o la alteración de las glándulas de Meibomio (5).

Curiosamente, no se pudo encontrar en dicho estudio ninguna relación entre los valores de AVF y los de gravedad de tinción con fluoresceína en los pacientes con SSJ. El estudio sugería que las lesiones corneales que afectaban al eje visual parecían tener mayor impacto en los valores de AVF que la tinción punteada. Una superficie corneal irregular resultante de un déficit lagrimal ha mostrado estar asociada con una pobre calidad visual (5).

Un reciente estudio con un nuevo sistema no invasivo de análisis de la estabilidad lacrimal (TSAS — tear stability analysis system-), que evalúa ésta mediante imágenes dinámicas de videokeratoscopio de la película lagrimal capturadas continuadamente cada segundo durante 10 segundos, empleando índices topográficos de regularidad y asimetría de la superficie corneal, reveló una degradación significativa de la estabilidad cinética lagrimal en los pacientes con ojo seco, con empeoramiento de dichos índices con el tiempo. Dicho estudio también mostró mejoría de los índices de regularidad y asimetría de la superficie en los pacientes con ojo seco a los que se les ocluía el punto lagrimal con tapones (6). A pesar de que no se realizaron las mediciones de TSAS en ese estudio, el empeoramiento de los índices de regularidad y asi- metría de la superficie corneal a lo largo del tiempo podría resultar en la disminución de los valores de AVF observada en los pacientes con ojo seco.

Los hallazgos de que la AVF mejoraba en los pacientes que recibían oclusión puntal podrían explicarse por la mejoría en dichos índices topográficos.

Una medición simultánea del TSAS dinámico y la AVF en los pacientes con ojo seco sería esencial y proporcionaría una información muy interesante. Además, estudios que se centraran en las correlaciones entre los valores de AVF y las escalas del Instituto Oftalmológico Nacional (NEI — National Eye Institute-) o del Índice de Enfermedad de la Superficie Ocular (OSDI -Ocular Surface Disease Index-) también proporcionarían información inestimable.

En resumen, el sistema de medición de la AVF es una tecnología nueva que no parece ser sólo una herramienta efectiva en la detección de cambios dinámicos de la AV en el ojo seco, en las alteraciones de la superficie ocular y en los sujetos normales, sino también un método prometedor para la evaluación de los resultados del manejo del ojo seco.

\section{BIBLIOGRAFÍA}

1. The definition and classification of dry eye disease: report of the Definition and Classification Subcomité of the International Dry Eye WorkShop (2007). Ocul Surf 2007; 5: 75-92.

2. Goto E, Yagi Y, Matsumoto Y, Tsubota K. Impaired functional visual acuity of dry eye patients. Am J Ophthalmol 2002; 133: 181-186.

3. Tanaka M, Takano Y, Dogru M, Toda I, Asano-Kato N, Komai-Hori $Y$, et al. Effect of preoperative tear function on early functional visual acuity after laser in situ keratomileusis. J Cataract Refract Surg 2004; 30: 2311-2315.

4. Ishida R, Kojima T, Dogru M, Kaido M, Matsumoto $Y$, Tanaka $M$, et al. The application of a new continuous functional visual acuity measurement system in dry eye syndromes. Am J Ophthalmol 2005; 139: 253-258.

5. Kaido M, Dogru M, Yamada M, Sotozono C, Kinoshita S, Shimazaki J, et al. Functional visual acuity in StevensJohnson syndrome. Am J Ophthalmol 2006; 142: 917-922.

6. Kojima T, Ishida R, Dogru M, Goto E, Takano Y, Matsumoto $Y$, et al. A new noninvasive tear stability analysis system for the assessment of dry eyes. Invest Ophthalmol Vis Sci 2004; 45: 1369-1374. 